\title{
Semipurified Rhamnolipid Mixes Protect Brassica napus Against Leptosphaeria maculans Early Infections
}

\author{
Noadya Monnier, ${ }^{1}$ Marion Cordier, ${ }^{2}$ Abdellatif Dahi, ${ }^{1}$ Valérie Santoni, ${ }^{1}$ Stéphanie Guénin, ${ }^{3}$ Christophe Clément, ${ }^{2}$ \\ Catherine Sarazin, ${ }^{4}$ Annette Penaud, ${ }^{5}$ Stephan Dorey, ${ }^{2}$ Sylvain Cordelier, ${ }^{2}$ and Sonia Rippa ${ }^{1, \dagger}$ \\ ${ }^{1}$ Unité de Génie Enzymatique et Cellulaire, UMR CNRS 7025, Sorbonne Universités, Université de Technologie de Compiègne, SFR Condorcet \\ FR CNRS 3417, Compiègne, France \\ 2 Unité Résistance Induite et Bioprotection des Plantes, EA 4707, Université de Reims Champagne Ardenne, SFR Condorcet FR CNRS 3417 , \\ Reims, France \\ ${ }^{3}$ Centre de Ressources Régional en Biologie Moléculaire, Université de Picardie Jules Verne, SFR Condorcet FR CNRS 3417, Amiens, France \\ ${ }^{4}$ Unité de Génie Enzymatique et Cellulaire, UMR CNRS 7025, Université de Picardie Jules Verne, SFR Condorcet FR CNRS 3417 , Amiens, \\ France \\ 5 Terres Inovia, Département de Génétique et Protection des Cultures-Phytopathologie, Thivernal-Grignon, France
}

Accepted for publication 23 December 2019.

\begin{abstract}
The rapeseed crop (Brassica napus) has to cope with fungal diseases that significantly impacts yields. In particular, the fungal pathogen Leptosphaeria maculans, the causal agent of blackleg disease (also named Phoma stem canker), is a worldwide issue to this crop. Considering environmental concerns, it is essential to propose alternative natural compounds for rapeseed crop protection to reduce chemical fungicide use. Here we report data showing the efficacy of semipurified rhamnolipid (RL) mixes from bacterial origin to protect rapeseed against L. maculans at early stages of infection in controlled conditions. In addition, we show that RL solutions have excellent adhesion properties when sprayed onto rapeseed leaves, without adding
\end{abstract}

ABSTRACT any adjuvant. We demonstrate that RL mixes display direct antimycelial properties against the pathogen and stimulate plant defense responses in rapeseed. Our results validate, a preventive action of low RL concentrations to protect rapeseed against $L$. maculans and a curative effect in specific conditions when applied after the inoculation of the pathogen spores. Semipurified RL mixes therefore appear to be real cost-effective compounds that could be used in fields as biocontrol products to fight L. maculans early infections of rapeseed.

Keywords: antifungal, biological control, canola, disease control and pest management, elicitor, oilseed rape, plant immunity
Phoma stem canker (blackleg) is an internationally important disease of Brassica crops, especially Brassica napus, which is the second oilseed crop production in the world (Carré and Pouzet 2014). It causes serious yield losses in Europe, North America, and Australia. Globally, Phoma stem canker causes approximately $€ 800$ million worth of yield losses, making it a considerable threat to sustainable rapeseed production (Fitt et al. 2006). The causal agent, Leptosphaeria maculans (anamorph Phoma lingam), is a fungus of the phylum Ascomycota. L. maculans airborne ascospores (sexual spores), released from pseudothecia developed on woody residues of infected plants, are the most common primary inoculum (West et al. 2001). They are scattered after wetting by rain or dew, over long distances, and can remain viable on inert materials for more than 200 days even in the absence of a host (Barua et al. 2017). After germination, depending on temperature and wetting conditions, L. maculans spreads via stomata and wounds, causing cotyledon or

\section{${ }^{\dagger}$ Corresponding author: S. Rippa; sonia.rippa@utc.fr}

Funding: This research was funded in the context of the EliZa project by the SAS PIVERT, within the frame of the French Institute for the Energy Transition (Institut pour la Transition Energétique [ITE] PIVERT) as part of the Investments for the Future ("Investissements d'Avenir"), by the French Government under the reference ANR-001-01. The Hauts-de-France council and European Regional Development Fund also co-founded the equipment used in UTC and UPJV within CPER 20072020 .

*The $e$-Xtra logo stands for "electronic extra" and indicates that four supplementary tables are published online.

The author(s) declare no conflict of interest.

(c) 2020 The American Phytopathological Society leaf spots primary infections visible as chlorotic tissue surrounding the infection. These spots develop on young susceptible plants in lesions containing pycnidia that produce conidia (asexual spores) and can cause secondary infections over shorter distances through rain splashes (Fitt et al. 2006). Fungus hyphae develop down petioles to reach the stem to produce the most damaging stem cankers at the stem base (crown canker) (West et al. 2001). L. maculans, described as an hemibiotroph (Oliver 2012), has a complex life cycle in the host plant, starting with a biotrophic stage in the cotyledons and young leaves, followed by an endophytic stage when growing in the stem. Finally, a necrotrophic stage leads to the formation of canker. Several control measures such as growing resistant cultivars (qualitative and quantitative resistances), crop rotation, stubble management and fungicides are used to reduce the development of disease (Marcroft et al. 2012; West et al. 2001). These measures depend on the agricultural practices, the climate, the disease severity, and the differences in the economics of rapeseed production (West et al. 2001). However, L. maculans has a very high evolutionary potential that renders fungicides and rapeseed genetic resistances rapidly ineffective (Sewell et al. 2017; Sprague et al. 2006).

Rhamnolipids (RLs) are natural glycolipids produced by Pseudomonas and Burkholderia bacteria, and mainly by the bacteria Pseudomonas aeruginosa. They are amphiphilic compounds composed of one (monoRLs) or two (diRLs) rhamnose glycosyl polar heads linked through a beta-glycosidic bond to one or two 3-hydroxyfatty acid hydrophobic tails (from 8 to 16 carbons, sometimes with an unsaturation) (Abdel-Mawgoud et al. 2010). In bacteria, they are involved in surface motility and biofilm development (Abdel-Mawgoud et al. 2010). RLs are well known as biosurfactant used for a wide range of industrial applications 
(Rikalović et al. 2015). In the last 10 years, they have also been proposed for agronomical purposes (Chen et al. 2017). They are considered as a very promising biocontrol solution for crop protection since they can be produced from agricultural waste materials (Tan and Li 2018), they are biodegradable (Mohan et al. 2006), have a low ecotoxicity profile (Johann et al. 2016) and display foliar penetration and wettability properties (Liu et al. 2016). The high production costs of RLs puts a brake on an agronomic use and important efforts are made to reduce them (Henkel et al. 2012).

RLs have antimicrobial properties and stimulate plant immunity (Varnier et al. 2009). Their direct antifungal properties on spores and mycelium are proposed to be linked to their ability to intercalate into fungal cell membrane, potentially leading to their disruption (Monnier et al. 2019; Stanghellini and Miller 1997). Such a general mode of action should limit resistance emergence and is very promising to reduce the use of chemical fungicides. In plants, a mix of purified mono and diRLs with fatty acyl chain length of 10 carbons (C10 RLs) stimulate defense and protection mechanisms. Indeed, they are known to trigger an immune response in Arabidopsis characterized by signaling molecules accumulation and defense gene activation (Sanchez et al. 2012). This dual mode of action protects rapeseed leaf tissues from the opportunistic fungus Botrytis cinerea (Monnier et al. 2018). It is now necessary to demonstrate the efficiency of semipurified RL mixes to protect annual plants of drastic phytosanitary problems to study the economic reality of their agronomic potential. Determining if RL mixes can act as preventive and/or curative agents toward phytopathogenic fungi also becomes a significant purpose. This is necessary to consider their applicative interest as a part of biocontrol solutions before field trials.

Here we report a study on rapeseed protection by RL mixes against $L$. maculans. Two semipurified commercial RL mixes were analyzed and used to evaluate their protective efficacy on young rapeseed seedlings known as the most susceptible stage of disease development. The seedlings were sprayed with RL mixes, before, simultaneously or after the pathogen inoculation. Direct antifungal activities of RL mixes on L. maculans were also investigated. Quantification of gene expression and callose deposition were performed to characterize defense and protection mechanisms induced.

\section{MATERIALS AND METHODS}

Biological material. Four winter rapeseed cultivars were used. Basalti seeds were graciously donated by the seed company Caussade semences (Caussade, France). Darmor- $b z h$ was provided by BrACySol Resource Center (INRA, France). Anastasia and Archimedes cultivars were obtained from the Groupement National Interprofessionnel des Semences (GNIS, France); both cultivars are from Limagrain (France). Basalti is described as being a sensitive cultivar to phoma. Darmor-bzh and Anastasia are known to harbor quantitative resistances. Archimedes is a qualitative resistant cultivar harboring the resistance gene $\operatorname{Rlm} 7$ (personal communication of the technical center Terres Inovia (Grignon, France)). Rapeseed was grown in soil in controlled conditions $(12 \mathrm{~h}$ photoperiod, $20^{\circ} \mathrm{C}$ day $/ 19^{\circ} \mathrm{C}$ night, $60 \mu \mathrm{mol} \mathrm{m}^{-2} \mathrm{~s}^{-1}$ light intensity, $60 \%$ relative humidity). Leptosphaeria maculans strain FEM66, isolated from rapeseed was provided by Terres Inovia. It harbors the AvrLml and the AvrLm4 avirulence genes. The fungus was grown by transferring a piece of agar containing mycelium on tomato juice agar medium ( $25 \%$ tomato juice, vol/vol, 3.75\% agar, wt/vol), and incubated at $18^{\circ} \mathrm{C}$ in the dark. To get spores, plates were placed at $25^{\circ} \mathrm{C}$ with $12 \mathrm{~h}$ photoperiod in a climatic chamber enlightened by one fluorescent and three white light tubes at $30 \mu \mathrm{mol} \mathrm{m} \mathrm{m}^{-2} \mathrm{~s}^{-1}$ light intensity. Spores were collected with sterile water $(4 \mathrm{ml})$ from 2week-old culture plates. Spore concentration was determined with a Malassez cell, before dilution with sterile water to obtain a $10^{6}$ spores $\mathrm{ml}^{-1}$ concentration.
RL mix solutions. Two commercial RL mixes containing RLs (90\%) from Pseudomonas aeruginosa fermentations were used. The mix named RL90-A was provided by AGAE Technologies (Corvalis, U.S.A.) (reference R90). The mix named RL90-N, from NatSurFact (Fairfax, U.S.A.) (reference 90LCBS), was kindly provided by SAS Pivert (Compiègne, France). Both RL mixes were diluted in deionized water for all experiments, except for mass spectrometry analysis.

HPLC-ESI-QTOF analysis. RL mix compositions were analyzed using ultra high-pressure liquid chromatography (UHPLC 1290 Infinity) coupled with high resolution-mass spectrometry (HR-MS QTOF UHD 6538) from Agilent Technologies. In brief, $2 \mu \mathrm{l}$ of $10 \mathrm{mg} \mathrm{ml}^{-1}$ of each RL mix in methanol were injected and the compounds were separated on a Thermo Hypersil Gold C18 column $(2.1 \times 100 \mathrm{~mm}$; particle size $1.9 \mathrm{~mm})$ coupled to an appropriate guard column. The column temperature was set to $50^{\circ} \mathrm{C}$ and the flow rate to $0.4 \mathrm{ml} \mathrm{min}-1$. The separation gradient was generated using $0.1 \%$ formic acid in water (solvent $\mathrm{A}$ ) and acetonitrile (solvent B). A gradient elution was performed initially with $90 \%$ A and $10 \% \mathrm{~B}$ for $1 \mathrm{~min}$, then with $40 \% \mathrm{~A}$ and $60 \% \mathrm{~B}$ for $20 \mathrm{~min}$, and finally with $100 \%$ B for $10 \mathrm{~min}$. The column was cleaned with $100 \%$ $\mathrm{B}$ for $5 \mathrm{~min}$. Mass spectra were acquired using a dual electrospray ionization in negative-ion mode. The source temperature was set up at $300^{\circ} \mathrm{C}$. The nebulization gas, the ion spray voltage and the fragmentor were adjusted to 30 pound-force per square (psi), $3.8 \mathrm{kV}$ and $100 \mathrm{~V}$, respectively. The range of mass detected on time of flight extended from $100 \mathrm{~m} / \mathrm{z}$ to $1700 \mathrm{~m} / \mathrm{z}$, with 1 scan per second. All data were acquired using auto-MSMS mode, controlled and processed by the MassHunter B.07 software. The composition of the different RLs were expressed as a relative percentage of their peak areas with respect to the total peak areas of all the detected features by dividing each RL peak intensity by the sum of all detected features in the sample and multiplying by 100 . The analyses were performed twice, with similar results. Presented values correspond to one representative experiment.

Conductimetry measurements. Conductivity of RL solutions was measured using COND LAQUAtwin conductimeter (Model B-771, Horiba, Kyoto, Japan) at room temperature (23 \pm $\left.2^{\circ} \mathrm{C}\right)$. After a calibration with a conductivity standard $\left(1.41 \mathrm{mS} \mathrm{cm}^{-1}\right.$ at $25^{\circ} \mathrm{C}$, VWR Chemicals, Fontenay-sous-bois, France), $100 \mu \mathrm{l}$ of sample was placed in the measuring cell for conductivity determination. Reported values are the mean of at least three independent measurements.

Contact angle measurements. Contact angle measurements were conducted with a Krüss DSA $10 \mathrm{Mk} 2$ goniometer (Germany) at room temperature $\left(23 \pm 2{ }^{\circ} \mathrm{C}\right)$. Leaf surfaces (adaxial side, $3-\mathrm{cm}$ segments) from 6-week-old rapeseed plants of Darmor- $b z h$ cultivar were fixed on a double-sided tape, mounted beforehand on glass slides. For each measurement, a $5 \mu \mathrm{l}$ drop (water or RL solutions) was formed at the tip of the syringe. After placing the drop onto the leaf surface away from leaf veins, the contact angle was measured within 3 min by a sessile drop method (Bejarano et al. 2017). Each reported contact angle is the mean of at least five independent measurements.

Protection experiments. Treatments were performed by spraying water (control conditions) or RL mix solutions on 12- to 21-day-old seedlings placed in mini-greenhouses. Spore inoculations were adapted from Pongam et al. (1998) and were performed 14 days after sowing. Each cotyledon was slightly wounded in the middle with a $10 \mu \mathrm{l}$ pipet tip and inoculated with $10 \mu \mathrm{l}$ of conidia solution. Seedlings were then placed into darkness for 2 days before being transferred in the growth chamber. Lesion measurements were realized 28 days after sowing. Two perpendicular lesion diameters were measured $\left(D_{1}\right.$ and $\left.D_{2}\right)$, and the lesion area $\left(A_{L}\right)$ was then calculated using Equation 1. Results are means of at least three independent experiments performed on a minimum of 10 plants per condition corresponding each to 20 lesions per condition. For the dose treatment assays, due to space constraints, four conditions 
were always performed at the same time, including the control; both RL solution effects were tested separately.

$$
A_{L}=\pi \frac{D_{1} x D_{2}}{4} \mathrm{M}
$$

Measurements of mycelium radial growth. Mycelium plugs of $5.5 \mathrm{~mm}$ diameter were excised from 2-week-old tomato juice agar plates of $L$. maculans. They were transferred on new petri dishes (diameter $9 \mathrm{~cm})$ containing V8 agar medium $(16 \% \mathrm{~V} 8$ juice, vol/vol, $2.4 \mathrm{~g} \mathrm{liter}^{-1}$ of $\mathrm{CaCO}_{3}$, and $16 \mathrm{~g} \mathrm{liter}^{-1}$ of agar) supplemented with RLs when necessary. Three plates were prepared for each condition. For each plate, mycelium radial growth was determined by measuring two perpendicular diameters after 14 days. Presented values are means of three independent experiments (nine plates for each condition).

Gene expression quantifications. Rapeseed cotyledons were harvested 24 and $48 \mathrm{~h}$ after treatment and immediately frozen in liquid nitrogen. Tissues were kept at $-80^{\circ} \mathrm{C}$ until RNA extractions, realized with the RNeasy plant mini kit (Qiagen, Hilden, Germany), with RNase free DNase set (Qiagen). RNA concentration and purity were determined and checked with a NanoDrop 1000 spectrophotometer (ThermoFisher Scientific, Waltham, MA, U.S.A.). Reverse transcriptions were performed using RevertAid Reverse transcription (ThermoFisher) on $4 \mu \mathrm{g}$ of RNA. Quantitative PCR were carried out using SYBR green master mix and a LightCycler 480 (Roche, Bâle, Switzerland) according to (Monnier et al. 2018). Expression quantification of $B n W R K Y 33, B n P R 4, B n P R l$, and $B n E R F 1$ genes were performed. Reference gene $B n E F 1 a$ was chosen among three tested genes (BnELF5, BnGDII, and BnEFla) for its stability in our conditions. All primers were synthesized by Eurofins Genomics (Luxembourg) and their sequences are presented in Supplementary Table S1. The results are means of four independent experiments (12 plants for each condition). CT (crossing threshold) and PCR efficiency (E) values were used to calculate expression using the formula $\mathrm{E}_{\mathrm{T}}^{\left(\mathrm{CT}_{\mathrm{C}}-\mathrm{CT}_{\mathrm{RL}}\right)} / \mathrm{E}_{\mathrm{R}}^{\left(\mathrm{CT}_{\mathrm{C}}-\mathrm{CT}_{\mathrm{RL}}\right)}$, where $\mathrm{T}$ is the target gene, $\mathrm{R}$ is the reference gene BnEFla, C refers

TABLE 1. Global composition of rhamnolipid (RL) mixes used in the study, determined by HPLC-ESI-QTOF analysis

\begin{tabular}{lccc}
\hline Mix & $\begin{array}{c}\text { Total RL } \\
\text { content }\end{array}$ & $\begin{array}{c}\text { MonoRL content } \\
\text { (\% of total RL } \\
\text { content) }\end{array}$ & $\begin{array}{c}\text { DiRL content } \\
\text { (\% of total RL } \\
\text { content) }\end{array}$ \\
\hline RL-A & $85 \%$ & $66 \%$ & $34 \%$ \\
RL-N & $59 \%$ & $41 \%$ & $59 \%$ \\
\hline
\end{tabular}

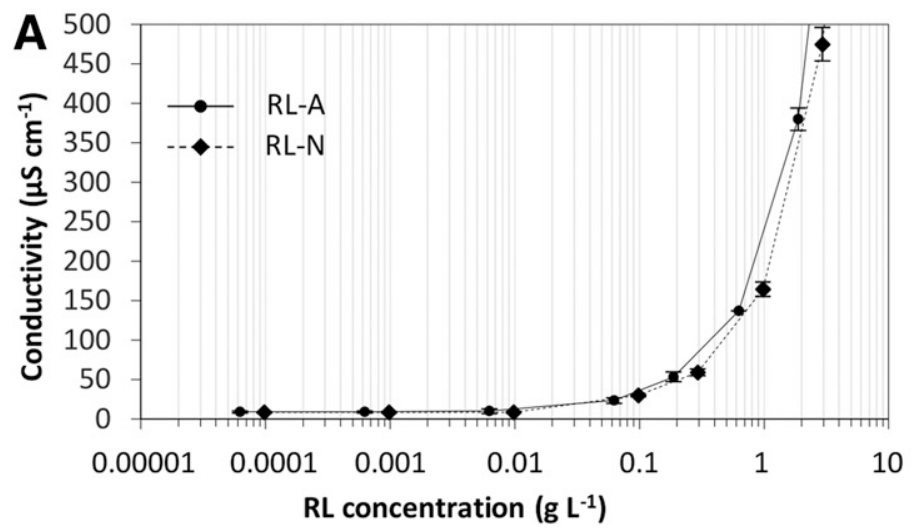

to cDNA from the control plants, and RL refers to cDNA from the treated plants. The relative expression corresponded to the value obtained for RL-treated plants relative to the value obtained for control plants, according to Guénin et al. (2009).

Callose measurements. Cotyledons from 12-day-old seedlings were cut $24 \mathrm{~h}$ after a spray treatment with water or RL solutions. The staining of callose was made using methyl blue as described in (Monnier et al. 2018). Callose was quantified from photographs using ImageJ software as the number of callose corresponding pixels relative to the total number of pixels covering plant material. Contrast settings of the photographs were adjusted to obtain an optimal separation of the callose signal from the background signal. For each condition, four cotyledons (32 photographs) were analyzed. The results are means of three independent repetitions (96 photographs from 12 cotyledons for each condition).

Statistical analysis. Statistical differences in the protection assays, defense gene expression and callose accumulation quantifications, were analyzed on R software (R Core Team 2014) using Kruskal-Wallis with post hoc tests $(P<0.05)$ (kruskalmc function of pgirmess package [Giraudoux et al. 2018]). A $t$ test analysis was performed in inhibition of mycelium growth assays.

\section{RESULTS}

Characterization of RL mixes. RLs are naturally produced as a mixture of various compounds depending on different factors, including the $P$. aeruginosa strain and the carbon source used for production (Rikalovic et al. 2013). Besides, producers of commercial RL mixes indicated that the composition depends on product batch (personal communication with providers). HPLC-ESI-QTOF analysis were performed in order to characterize the mixes used in this study. The purity of the mixes were estimated around 85 and $59 \%$ of RLs for RL-A and RL-N, respectively. In both mixes, mono and diRL were identified. MonoRL were the major RLs present in RL-A, while diRL were the major RLs in RL-N (Table 1). Further characterization of RL content showed that most identified RLs had a hydrophobic part composed of two saturated carbon chains ranging from $\mathrm{C} 8$ to C12 (Supplementary Table S2). In both mixes, the two major compounds were mono and diRL with two saturated C10 fatty acid chains. RLs being amphiphilic compounds, they spontaneously aggregate in water. Critical aggregation concentration (CAC) was then evaluated by conductimetry, as previously reported (Özdemir et al. 2004). Similar results were obtained with RL-A and RL-N. The conductivity started to increase when RL concentration was higher than $0.1 \mathrm{~g} \mathrm{liter}^{-1}$ corresponding to a CAC of around $0.1 \mathrm{~g} \mathrm{liter}^{-1}$ for both mixes (Fig. 1A).

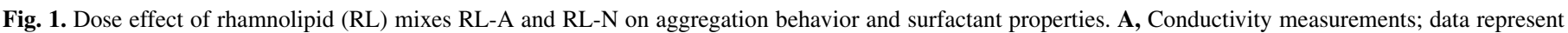

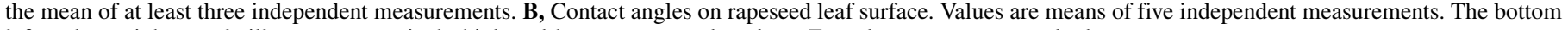
left and top right panels illustrate respectively high and low contact angle values. Error bars represent standard error.

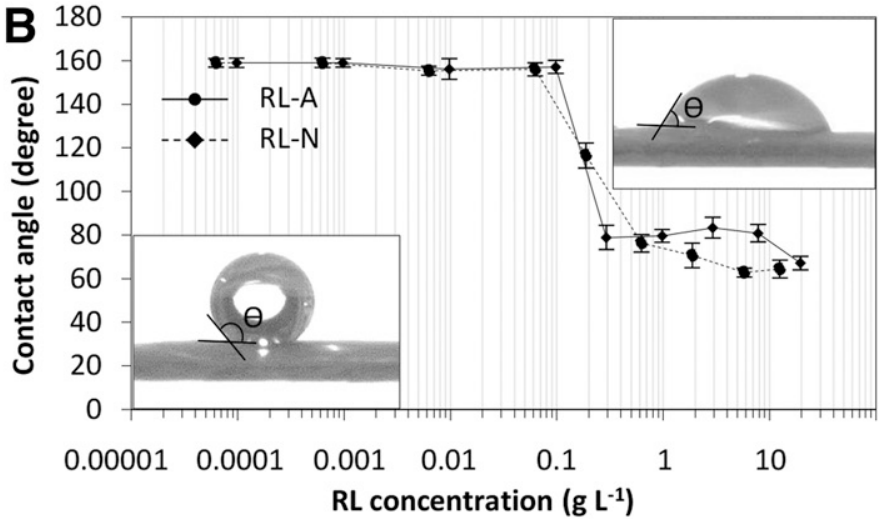


Surfactant properties of RL mixes on rapeseed leaf surface. Rapeseed foliar cuticles are highly hydrophobic. Accordingly, the contact angle between water and the adaxial face of rapeseed leaves was higher than $150^{\circ}$ (Fig. 1B). Also, the adhesion of water droplets on leaf surface appeared to be very low as droplet deposition was difficult, with a tendency to bounce. This was confirmed by roll off angle measurements realized with a tilting platform as performed by Bejarano et al. 2017. Values around $5^{\circ}$ were obtained for water droplets (Supplementary Table S3). RL solutions with a concentration higher than $0.1 \mathrm{~g} \mathrm{liter}^{-1}$ had clearly reduced contact angles as compared with water (Fig. 1B). The surfactant properties of both RL mixes allowed, in the same way, a significantly better adhesion for leaf surface. This was confirmed by the fact that the drops of RL solutions did not roll off when increasing the tilting angle (Supplementary Table S3).

RL-triggered protection of susceptible rapeseed cultivar against $\boldsymbol{L}$. maculans. The RL mix potential for rapeseed protection against $L$. maculans was evaluated on four different cultivars. Twelve-day-old seedlings were sprayed to runoff with water or RL solutions at $0.1 \mathrm{~g} \mathrm{liter}^{-1}$, corresponding to approximatively $100 \mu \mathrm{M}$ RLs, based on an estimated molar weight calculated from the composition characterization $\left(534.0 \mathrm{~g} \mathrm{~mol}^{-1}\right.$ for RL-A and 575.8 for RL-N and their respective purity, Table 1). This concentration order of magnitude has been previously reported to trigger a rapeseed leaf

A

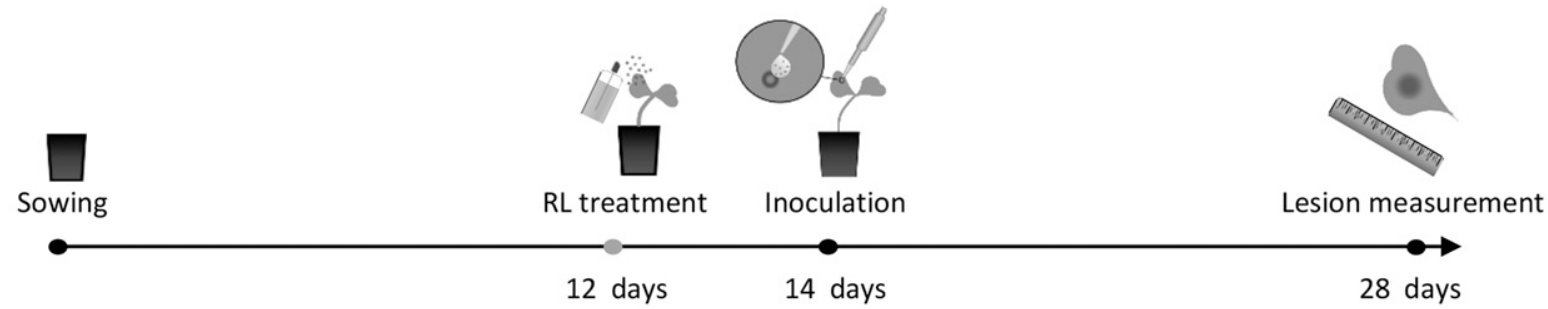

B

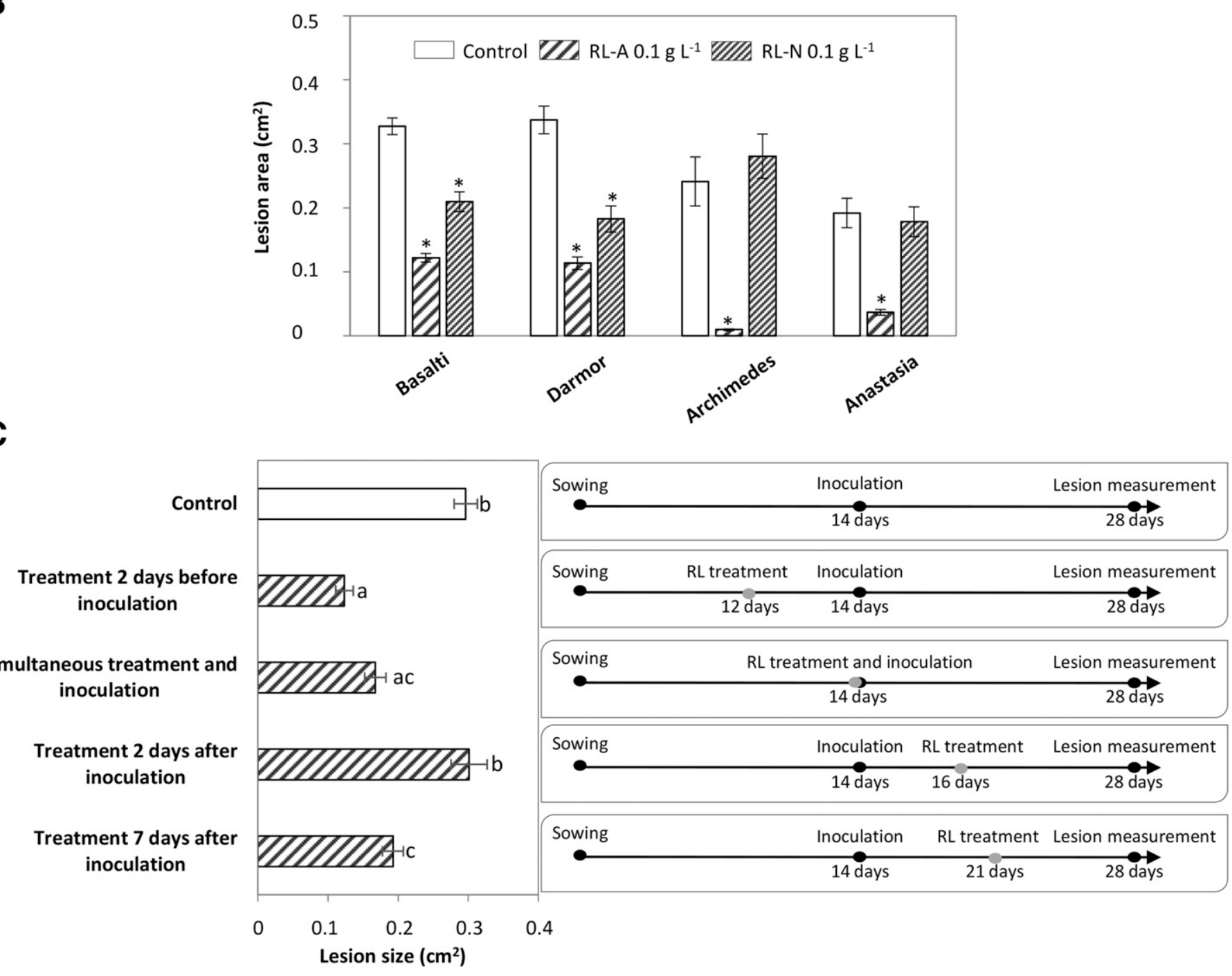

C

Fig. 2. Rhamnolipid (RL) mix-induced protection on rapeseed against Leptosphaeria maculans. A, Illustration of experimental protocol performed on different rapeseed cultivars. B, Lesion area quantification on control and $0.1 \mathrm{~g} \mathrm{liter}^{-1}$ of RL-A or RL-N treated cotyledons 2 days before inoculation in different cultivars. C, Lesion size observed on control or $0.1 \mathrm{~g} \mathrm{liter}^{-1}$ of RL-A treated cotyledons with different treatment methods. Presented data correspond to means of at least three biological repetitions, error bars correspond to standard errors and asterisks $(\mathbf{B})$ and different letters $(\mathbf{C})$ correspond to significant differences $(P<0.05)$ according to Kruskal-Wallis test. 
protection against B. cinerea (Monnier et al. 2018). Two days after treatment, conidia inoculations were performed and lesion sizes were measured 2 weeks later (Fig. 2A). In control plants, lesion sizes were higher on Basalti $\left(0.33 \pm 0.01 \mathrm{~cm}^{2}\right)$ and Darmor-bzh $\left(0.34 \pm 0.02 \mathrm{~cm}^{2}\right)$ than on Archimedes $\left(0.24 \pm 0.04 \mathrm{~cm}^{2}\right)$ and Anastasia cultivars $(0.19 \pm$ $0.02 \mathrm{~cm}^{2}$ ) (Fig. 2B). An important reduction in lesion size was observed on all cultivars treated with RL-A. Indeed, the average lesion size was $0.12 \pm 0.006 \mathrm{~cm}^{2}$ for Basalti (63\% size reduction), $0.11 \pm$ $0.01 \mathrm{~cm}^{2}$ for Darmor-bzh (66\% size reduction) and $0.04 \pm 0.004 \mathrm{~cm}^{2}$ for Anastasia ( $81 \%$ size reduction) while nearly no lesion was observed on Archimedes cultivar $\left(0.01 \pm 0.001 \mathrm{~cm}^{2}, 96 \%\right.$ size reduction). Basalti and Darmor- $b z h$ plants treated with RL-N also showed reduced lesion sizes with areas of $0.21 \pm 0.015 \mathrm{~cm}^{2}(37 \%$ size reduction) and $0.18 \pm$ $0.02 \mathrm{~cm}^{2}$ (46\% size reduction), respectively. In these conditions, no significant protection effect was observed on Archimedes and Anastasia cultivars (Fig. 2B). Therefore, RL mixes (especially RL-A, in a lesser extend RL-N) trigger an effective preventive protection in susceptible cultivars when treatment occurs 2 days before L. maculans infection.

Preventive and curative effects of $R L$ mixes against L. maculans. Cotyledons of sensitive Basalti seedlings were then treated with RL-A at $0.1 \mathrm{~g}$ liter $^{-1} 2$ days before $L$. maculans spore inoculation, or simultaneously to inoculation, or 2 and 7 days postinoculation (dpi) (Fig. 2C). Compared with the untreated control plants, smaller lesions were observed in pretreated cotyledons (lesion size of $0.12 \pm 0.01 \mathrm{~cm}^{2}, 60 \%$ of size reduction), in simultaneously RL-treated and spore-inoculated cotyledons $(0.17 \pm 0.01,43 \%$ of size reduction), and in 7 dpi-treated cotyledons (lesion size of $0.19 \pm 0.01 \mathrm{~cm}^{2}, 34 \%$ size reduction). On the contrary, RL-A treatment performed 2 dpi did not induce any significant difference on lesion size compared with the control condition. These results show preventive and curative effects of RL mixes on rapeseed against $L$. maculans lesion development that depend on the application time.

Concentration effects of $\mathrm{RL}$ mixes on rapeseed protection against $L$. maculans. Basalti cotyledons from 12-day-old seedlings were then treated with water or RL solutions 2 days before spore inoculation of $L$. maculans. For RL-A, the most efficient dose was $0.025 \mathrm{~g} \mathrm{liter}^{-1}$, with a lesion size of $0.10 \pm 0.01 \mathrm{~cm}^{2}$ (nearly $70 \%$ size reduction compared with the control) (Fig. 3A and C). The other concentrations of RL-A also induced a significant reduction of lesion size (about 40 to 50\% size reduction) (Fig. 3A). For RL-N solutions, concentrations from 0.025 to $0.1 \mathrm{~g} \mathrm{liter}^{-1}$ led to about 30 to $50 \%$ lesion size reduction (Fig. $3 \mathrm{~B}$ and $\mathrm{D}$ ). These results evidence efficiency of both RL mixes to protect rapeseed even at low concentrations. At $0.5 \mathrm{~g} \mathrm{liter}^{-1}$, the RL-N solution induced only a weak lesion size reduction.
A
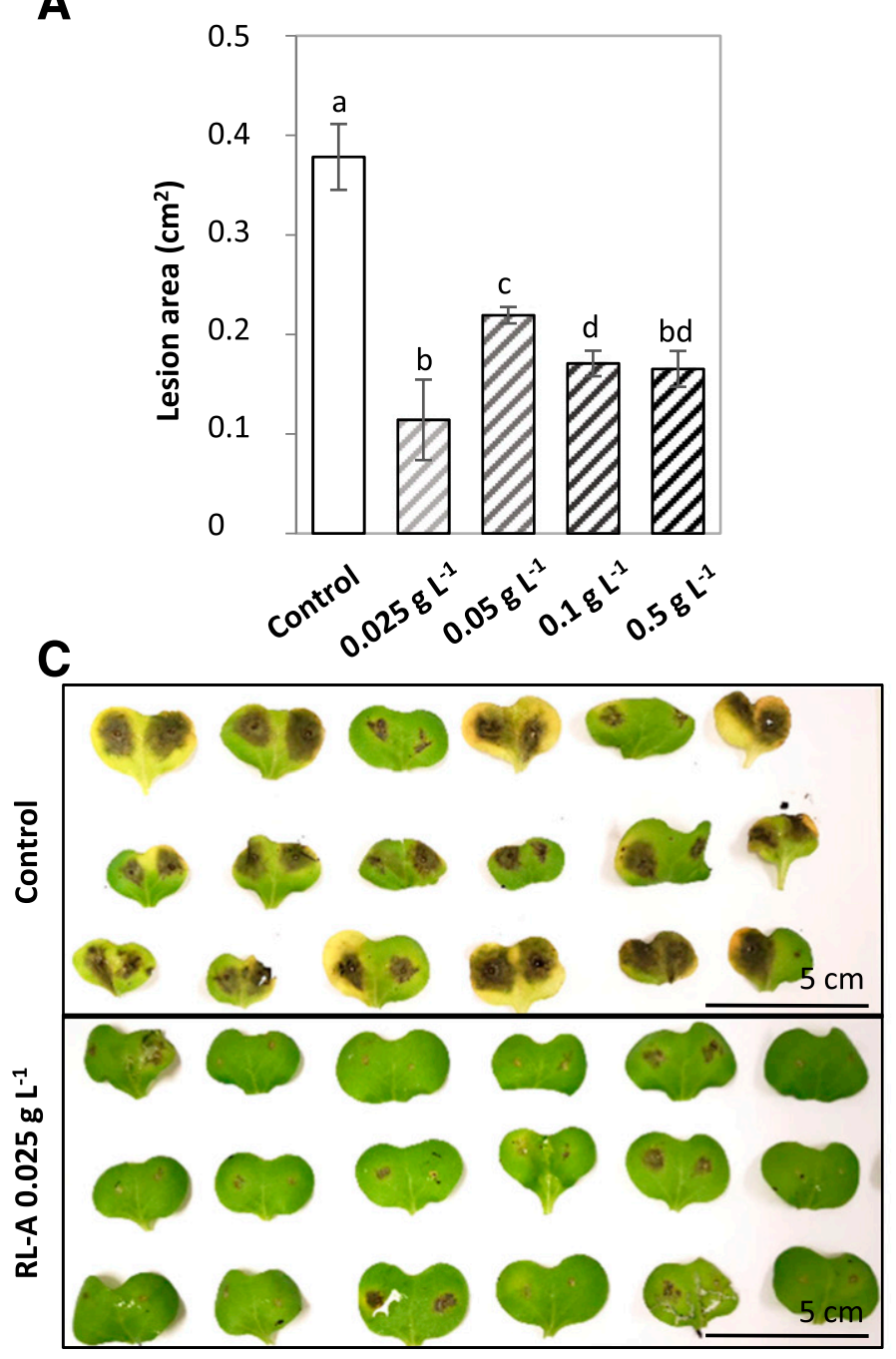

B

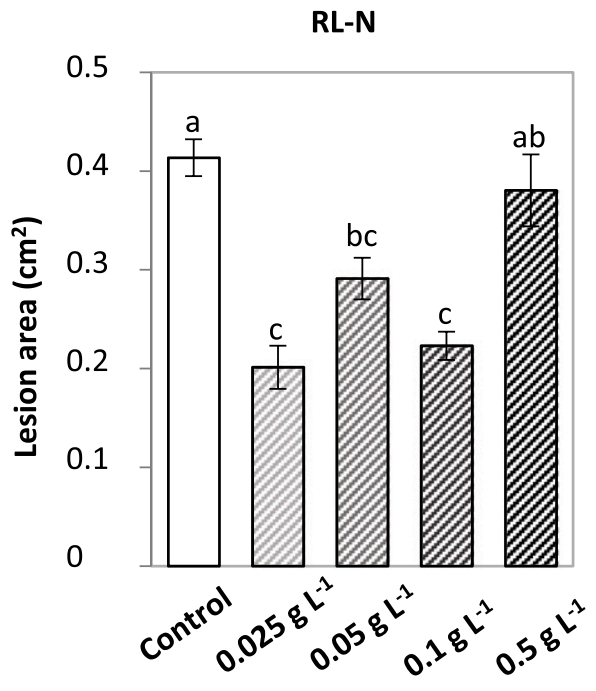

D

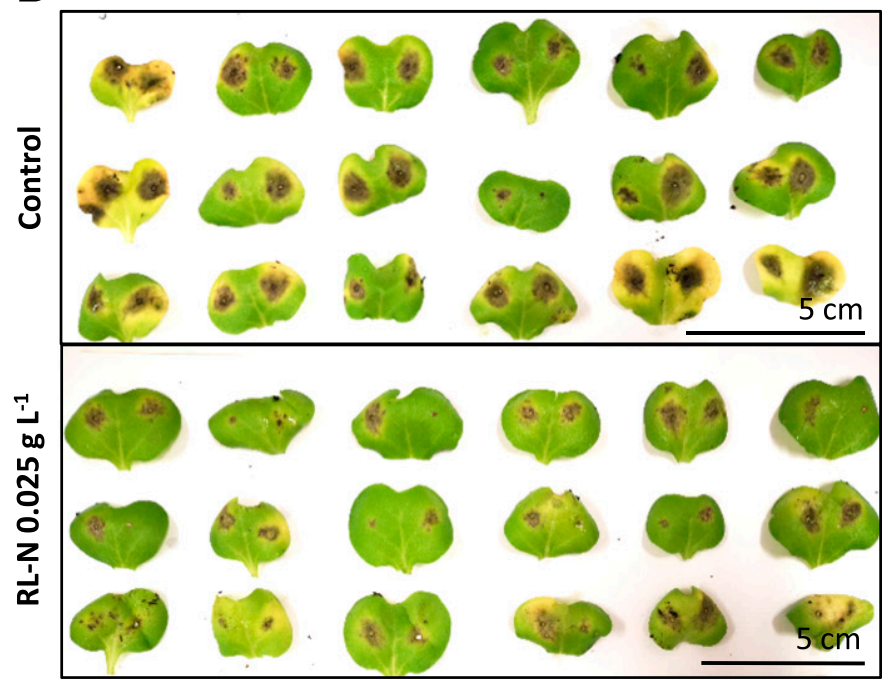

Fig. 3. Lesion size observed on control or rhamnolipid (RL) mix-treated Basalti cotyledons at different concentrations two days before $L$. maculans inoculation. A, RL-A, B, RL-N. Presented data correspond to mean of at least three biological repetitions, error bars correspond to standard errors and different letters correspond to significant differences $(P<0.05)$ according to Kruskal-Wallis test. Pictures of cotyledons treated with water or $0.025 \mathrm{~g}$ liter ${ }^{-1}$ of $\mathbf{C}$, RL-A and $\mathbf{D}$, RL-N, taken 14 days after inoculation. 
Direct antifungal activity of $R \mathbf{L}$ mixes. RL antifungal activities on $L$. maculans were then investigated. The effect of RL mixes was evaluated on $L$. maculans spore germination by nephelometry. No negative effect was observed on spore germination between control and RL-A or RL-N with concentrations ranging from 0.005 to $0.5 \mathrm{~g} \mathrm{liter}^{-1}$ (Supplementary Table S4). The effect of RL mixes on mycelium growth was also analyzed. Agar medium was supplemented with water or both RL solutions at different concentrations and inoculated with a $L$. maculans mycelium plug. An inhibition of mycelial growth was observed on medium plate supplemented with RL-A (Fig. 4A and C) and RL$\mathrm{N}$ (Fig. 4B and D) at every tested concentration except at $0.005 \mathrm{~g}$ liter $^{-1}$. The mycelium radial growth proportionally decreased with the RL mix concentration increase. The negative effect became significantly different from that of the control at $0.025 \mathrm{~g} \mathrm{liter}^{-1}$ of RL-A and 0.05 g liter $^{-1}$ of RL-N. At higher concentrations, RL-N appeared to be more efficient with a mycelial growth inhibition of $50 \%$ at $0.25 \mathrm{~g} \mathrm{liter}^{-1}$ and $66 \%$ at $0.5 \mathrm{~g} \mathrm{liter}^{-1}$, while RL-A reached $50 \%$ mycelial growth inhibition at $0.5 \mathrm{~g} \mathrm{liter}^{-1}$.

RL mix triggering of defense gene expression. RT-qPCR analysis were performed to assess the expression of defense genes previously described as markers of C10 RL perception in rapeseed (Monnier et al. 2018). Both RL mix solutions were sprayed at concentrations from 0.025 to $0.5 \mathrm{~g} \mathrm{liter}^{-1}$ on Basalti cotyledons of 12-day-old seedlings. RNAs were sampled 24 and $48 \mathrm{~h}$ following treatment. The $B n W R K Y 33$ gene expression was globally induced by both RL solutions. This overexpression was mostly stronger at $24 \mathrm{~h}$ compared with $48 \mathrm{~h}$ after treatment (Fig. 5A and B). The induction of BnPRl gene reached $41.6 \pm 20$ and $45.9 \pm 13$, respectively, at $0.1 \mathrm{~g} \mathrm{liter}^{-1}$ of RL-A mix and $0.5 \mathrm{~g} \mathrm{liter}^{-1}$ of RL-N mix at $24 \mathrm{~h}$ posttreatment (Fig. 5C and D). BnPR4 gene expression was also induced by both RL solutions with slight differences depending on the mix and the time (Fig. 5E and F). A low induction of the BnERF1 gene was also noticed (Fig. 5G and $\mathrm{H}$ ).

RL mix triggering of callose deposits. Callose deposits were also reported as a good protection marker induced by C10 RLs in rapeseed leaves (Monnier et al. 2018). Twelve-day-old Basalti cotyledons were sprayed with RL-A and RL-N mixes at concentrations ranging from 0.025 to $0.5 \mathrm{~g} \mathrm{liter}^{-1}$ and callose deposits were observed $24 \mathrm{~h}$ later. For both RL mixes, a slight augmentation of callose accumulation was observed at $0.025 \mathrm{~g}$ liter $^{-1}$ with relative callose intensities around 0.30 compared with $0.23 \pm 0.05$ for the control (Fig. 5I and J). The callose accumulation increased proportionally to RL concentration and became statistically different from the control at $0.05 \mathrm{~g} \mathrm{liter}^{-1}$ with relative callose intensities of $0.35 \pm 0.07$ and $0.38 \pm 0.05$ for RL-A and RL-N solutions, respectively. For RL-A, callose accumulation intensity increased up to the highest RL dose to reach the average value of $0.62 \pm 0.11$ at $0.5 \mathrm{~g} \mathrm{liter}^{-1}$ concentration. In response to RL-N treatment, a maximum of callose accumulation intensity was reached at $0.1 \mathrm{~g} \mathrm{liter}^{-1}$ with a value of $0.52 \pm 0.05$.

\section{DISCUSSION}

In this study, properties of two semipurified commercial RL mixes produced by $P$. aeruginosa were investigated for rapeseed protection against L. maculans. The spray of these RL solutions on cotyledons of whole rapeseed seedlings, 2 days before spore inoculation, led to a strong reduction of lesion sizes of L. maculans. In the nontreated conditions, variations of lesion sizes was observed
A
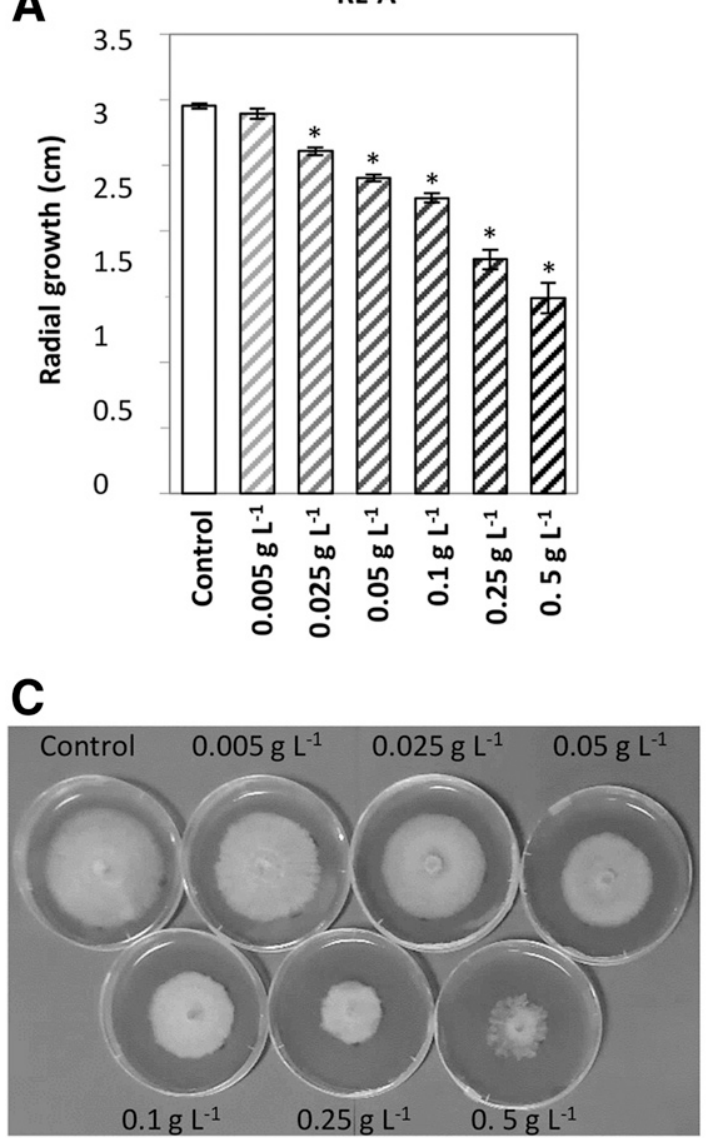

B

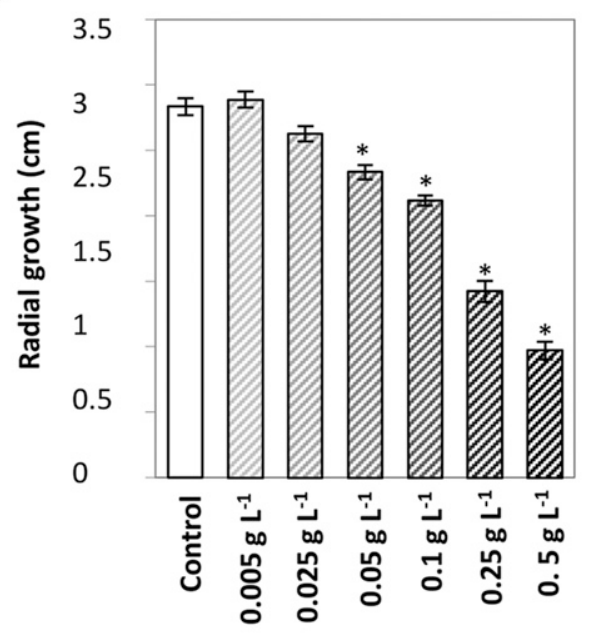

D

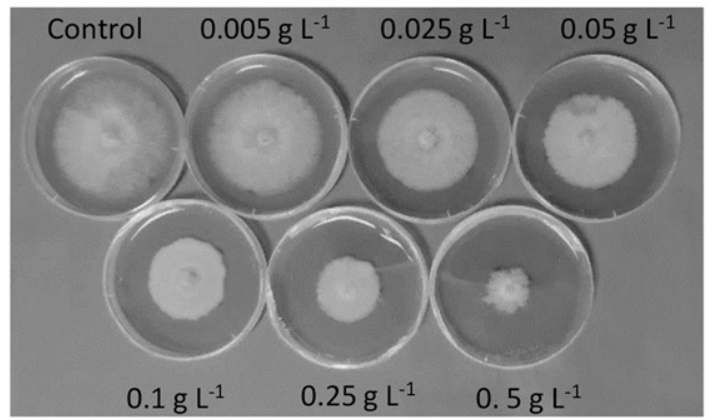

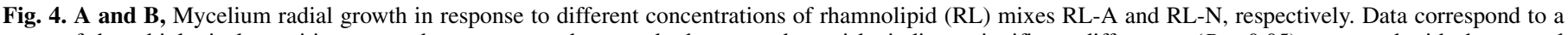

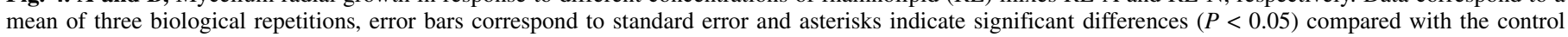

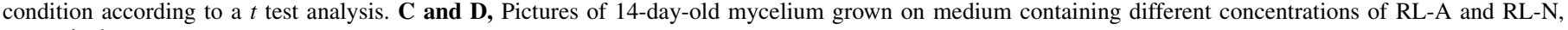
respectively. 

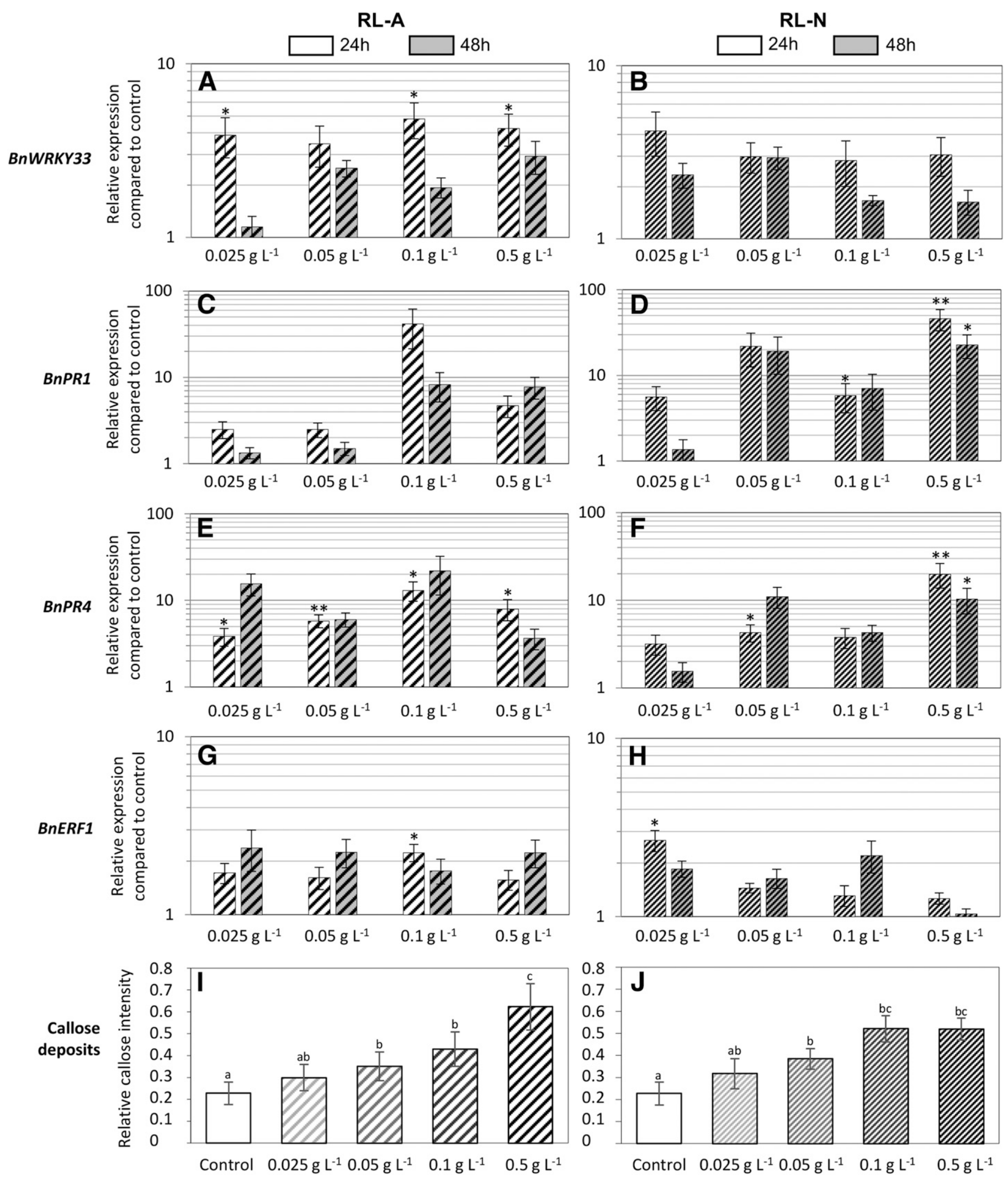

Fig. 5. Rhamnolipid (RL) mix-induced defense responses in Basalti cultivar. $\mathbf{A}$ and $\mathbf{B}, B n W R K Y 33, \mathbf{C}$ and $\mathbf{D}, B n P R 1, \mathbf{E}$ and $\mathbf{F}, B n P R 4$, and $\mathbf{G}$ and $\mathbf{H}, B n E R F 1$ gene expression were followed by RT-qPCR on rapeseed leaves at 24 and $48 \mathrm{~h}$ after treatment with the RL-A mix (A, C, E, and G) or the RL-N mix (B, D, F, and H). Data are the mean of three biological replicates showing the relative expression compared with the control conditions using the reference gene BnEF1 $\alpha$. Error bars correspond to standard error. Asterisks mark statistically significant conditions according to Kruskal-Wallis test $(*$ and $* *$ represent significant differences compared with the control condition and correspond respectively to $P<0.05$ and 0.01$)$. I and J, Callose deposits induced by respectively RL-A (I) and RL-N (J) mixes. The different letters correspond to significant differences $(P<0.05)$ according to Kruskal-Wallis test. 
among the four tested cultivars related to their susceptibility to L. maculans early infections. In treated conditions, both RL mixes were efficient to protect the cultivars Basalti and Darmor-bzh, highlighting the interest of using them to preventively protect susceptible rapeseed genotypes against $L$. maculans at early stages of development in compatible interactions. Even if they differ in their composition, both RL mixes showed antimycelial activity. They also induced defense gene expression, callose deposits and triggered rapeseed protection. It then appears that a precise distribution of RL species and a very high purity are not mandatory for RL mixes to protect rapeseed seedlings. These results bring out very interesting agronomical applications of RLs that could be developed with cost-effective mixes.

It should also be noticed that both mixes share very similar amphiphilic properties. Indeed, the CAC measured at $0.1 \mathrm{~g} \mathrm{liter}^{-1}$ corresponds to 100 to $150 \mu \mathrm{M}$ of different RL congener amounts approximatively in both RL mixes. This CAC value of the RL mix solutions is on the same order of magnitude as the CAC of purified RLs determined with the same method (Özdemir et al. 2004). These amphiphilic characteristics confer strong surfactant properties to the mixes, which is a major asset given the high hydrophobic cuticle of rapeseed leaves. Thus, RLs acting as wetting agents, RL applications were performed in our experiments without any addition of adjuvant compound or organic solvent to carry out the protection assays. Here again, our results show that a high purity and a precise composition are not required to get this very interesting applicative property of RLs.

Nevertheless, the total amount of RLs and some discrepancies in the fine composition of RLs could explain a global tendency to induce a better protection with the RL-A mix. Compared with RL$\mathrm{N}, \mathrm{RL}-\mathrm{A}$ contains a higher amount of RLs but also a higher global ratio of monoRLs to diRLs and a different distribution in the major RLs produced by $P$. aeruginosa. It is interesting to notice that the RL-A mix was efficient on the less susceptible cultivars Archimedes and Anastasia at the stage of development studied. This shows an interest of RL treatments to reinforce resistance in these conditions. Above all, the protection obtained with the two RL mixes demonstrates the robustness of the RL-triggered rapeseed protection against $L$. maculans.

It can reasonably be hypothesized that RL activity of plant defense induction is the main mode of action of RLs when they are applied 2 days before inoculation. This is supported by the induction of defense gene expression and callose deposits in the treated cotyledons 24 and $48 \mathrm{~h}$ after treatments. Interestingly, a recent transcriptomic analysis of the defense responses at the initial stage of the infection of susceptible B. napus by L. maculans shows an overexpression of WRKY defense associated transcription factors and the up-regulation of gene expression related to salicylic acid (SA) and jasmonic acid (JA) hormonal pathways (Haddadi et al. 2016). BnPRI and BnPR4 genes, triggered by RLs, are wellknown markers of the SA pathway and of the crosstalk between JA and ethylene pathways, respectively (Lebel et al. 1998; Thomma et al. 1998). BnWRKY33 overexpressing lines of B. napus display an overexpression of SA and JA marker genes (Wang et al. 2014). Also, WRKY33 is one of the highest overexpressed WRKY genes in resistant rapeseed cultivars infected by the fungus (Haddadi et al. 2019). Furthermore, the ability to rapidly develop a compact and continuous callose wall around the infection site is a characteristic of incompatible rapeseed/L. maculans interactions (Liu et al. 2018). Our data show that RL mixes induce efficient defense pathways to restrain $L$. maculans infection when they are preventively applied on rapeseed cotyledons. When treatment and inoculation are performed at the same time, defense responses are probably induced in the plant tissue during the time course of spore germination, and are strong enough to inhibit the fungus development. This is probably the main mode of action of RLs in this condition since RLs have no negative effect on the L. maculans spore germination.
The concentration effect studies reinforce the hypothesis of a mode of action mainly due to plant defense induction when the RL mixes are applied 2 days before spore inoculation. Indeed the $0.1 \mathrm{~g}$ liter $^{-1}$ concentration reduces the lesion area at about $50 \%$ of that of the control and has a negative effect on the mycelium growth (around $20 \%$ of mycelial growth inhibition). The $0.025 \mathrm{~g} \mathrm{liter}^{-1}$ concentration was also very efficient to protect rapeseed but had a weak effect on the mycelium development in growth medium plates. In the same way, the $0.5 \mathrm{~g} \mathrm{liter}^{-1}$ concentration of RL-N was the most efficient to inhibit L. maculans mycelium development in the mycelium inhibition study but was weakly efficient to protect rapeseed in the protection study. This last higher antimycelial effect of RL-N could be explained by a higher effect of diRLs than monoRLs from RL mix on different fungi as previously described (Sha et al. 2012). Altogether, our results show that there is not a correlation between a good antifungal activity and an effective plant protection. As observed in our RT-qPCR assays, both RL mixes have different effects on some defense genes. In particular, the $0.5 \mathrm{~g}$ liter $^{-1}$ concentration of RL-N triggers the highest BnPRI induction. Plant PR1 proteins have been recently proposed to inhibit cell death (Lincoln et al. 2018) and RLs are known to induce cell death in a concentration dependent manner (Monnier et al. 2018). The highest RL-N concentration appears here to promote associated cell death mechanisms and it could explain the weak protective effect observed toward L. maculans infections. Indeed, the plant defense mechanisms are very complex and fine-tuned. This must be especially true against $L$. maculans, which is a hemibiotrophic pathogen switching from a first biotrophic phase to a necrotrophic phase. An efficient disease protection probably implies to efficiently switch from defense responses adapted to a biotroph pathogen to defense responses adapted to a necrotroph pathogen (Haddadi et al. 2016, 2019).

In addition to preventive effectiveness, we also demonstrate for the first time, to our knowledge, that RLs can have a curative effect on a fungal pathogen in vivo, allowing the containment of the fungus growth even if they are applied after disease emergence in specific conditions. This curative result was observed when the RL mix spray was realized at $7 \mathrm{dpi}$, but not at 2 dpi. As much as the RL mix solutions have no inhibition effect on the spore germination, the curative effect at $7 \mathrm{dpi}$ could be due to the antimycelial properties of RLs, as supported by the mycelium growth inhibition observed at $0.1 \mathrm{~g} \mathrm{liter}^{-1}$. Since the greatest number of spot lesions of L. maculans are obtained with a leaf wetness duration of $48 \mathrm{~h}$ at $20^{\circ} \mathrm{C}$ in controlled conditions (Biddulph et al. 1999), it could be supposed that it is the optimal delay to get a complete spore germination on rapeseed leaf. However antimycelial activities could not fully explain the difference of RL effect observed at the two times of inoculation. Recently, a transcriptomic analysis of L. maculans virulence factors expressed during compatible interaction with $B$. napus seedlings showed that most of them are the most highly expressed at $7 \mathrm{dpi}$ (Sonah et al. 2016). Stimulating plant defense mechanisms with RLs at this time then appears very efficient to counteract the pathogen attack.

Overall, our results highlight the potential of RLs application to protect rapeseed preventively and curatively in specific conditions against $L$. maculans. The use of semipurified RL mixes, without any adjuvant, strengthens the economic feasibility to use them in field experiments. Our data also demonstrate the importance of studying application modalities of RL mixes on definite pathosystems before considering their use as biocontrol solution in integrated pest management strategies. In particular, to perform deeper investigations to study the early growth of the pathogen on plants treated by RLs would be very informative. Nevertheless, RL mixes, as already available commercial bioproducts with few purifying steps, were shown here to be very promising as a part of biocontrol strategies to reduce the use of chemical fungicides. 


\section{ACKNOWLEDGMENTS}

We thank L. Gutierrez (Centre de Ressources Régionales en Biologie Moléculaire, UPJV, Amiens) for his welcoming access to the molecular biology equipment; I. Pezron (Transformation Intégrée de la Matière Renouvelable, UTC, Compiègne) for her access to the goniometer and her help to perform contact angle measurements; T. Guillemet (Institut de Recherche en Horticulture et Semences UMR1345, Angers) to provide access to the nephelometry platform; and S. Acket (GEC laboratory) for his technical assistance to perform mass spectrometry analysis.

\section{LITERATURE CITED}

Abdel-Mawgoud, A. M., Lépine, F., and Déziel, E. 2010. Rhamnolipids: Diversity of structures, microbial origins and roles. Appl. Microbiol. Biotechnol. 86:1323-1336.

Barua, P., You, M. P., Bayliss, K. L., Lanoiselet, V., and Barbetti, M. J. 2017. Inert materials as long-term carriers and disseminators of viable Leptosphaeria maculans ascospores and wider implications for ascomycete pathogens. Plant Dis. 102:720-726.

Bejarano, A., Sauer, U., and Preininger, C. 2017. Design and development of a workflow for microbial spray formulations including decision criteria. Appl. Microbiol. Biotechnol. 101:7335-7346.

Biddulph, J. E., Fitt, B. D. L., Leech, P. K., Welham, S. J., and Gladders, P. 1999. Effects of temperature and wetness duration on infection of oilseed rape leaves by ascospores of Leptosphaeria maculans (stem canker). Eur. J. Plant Pathol. 105:769-781.

Carré, P., and Pouzet, A. 2014. Rapeseed market, worldwide and in Europe. OCL 21:D102.

Chen, J., Wu, Q., Hua, Y., Chen, J., Zhang, H., and Wang, H. 2017. Potential applications of biosurfactant rhamnolipids in agriculture and biomedicine. Appl. Microbiol. Biotechnol. 23-24:8309-8319.

Fitt, B. D. L., Brun, H., Barbetti, M. J., and Rimmer, S. R. 2006. World-wide importance of phoma stem canker (Leptosphaeria maculans and L. biglobosa) on oilseed rape (Brassica napus). Pages 3-15 in: Sustainable Strategies for Managing Brassica napus (Oilseed Rape) Resistance to Leptosphaeria maculans (Phoma Stem Canker). Springer-Verlag, Berlin, Heidelberg.

Giraudoux, P., Antonietti, J.-P., Beale, C., Pleydell, D., and Treglia, M. 2018. Package "pgirmess" Title Spatial Analysis and Data Mining for Field Ecologists. https://cran.r-project.org/web/packages/pgirmess/pgirmess.pdf

Guénin, S., Mauriat, M., Pelloux, J., Van Wuytswinkel, O., Bellini, C., and Gutierrez, L. 2009. Normalization of qRT-PCR data: the necessity of adopting a systematic, experimental conditions-specific, validation of references. J. Exp. Bot. 60:487-493.

Haddadi, P., Larkan, N. J., and Borhan, M. H. 2019. Dissecting R gene and host genetic background effect on the Brassica napus defense response to Leptosphaeria maculans. Sci. Rep. 9:6947.

Haddadi, P., Ma, L., Wang, H., and Borhan, M. H. 2016. Genome-wide transcriptomic analyses provide insights into the lifestyle transition and effector repertoire of Leptosphaeria maculans during the colonization of Brassica napus seedlings. Mol. Plant Pathol. 17:1196-1210.

Henkel, M., Müller, M. M., Kügler, J. H., Lovaglio, R. B., Contiero, J., Syldatk, C., and Hausmann, R. 2012. Rhamnolipids as biosurfactants from renewable resources: Concepts for next-generation rhamnolipid production. Process Biochem. 47:1207-1219.

Johann, S., Seiler, T. B., Tiso, T., Bluhm, K., Blank, L. M., and Hollert, H. 2016. Mechanism-specific and whole-organism ecotoxicity of monorhamnolipids. Sci. Total Environ. 548-549:155-163.

Lebel, E., Heifetz, P., Throne, L., Uknes, S., Rylas, J., and Ward, E. 1998. Functional analysis of regulatory sequences controlling $P R-1$ gene expression in Arabidopsis. Plant J. 16:223-233.

Lincoln, J. E., Sanchez, J. P., Zumstein, K., and Gilchrist, D. G. 2018. Plant and animal PR1 family members inhibit programmed cell death and suppress bacterial pathogens in plant tissues. Mol. Plant Pathol. 19:2111-2123.

Liu, F., Zou, Z., and Dilantha Fernando, W. G. 2018. Characterization of callose deposition and analysis of the callose synthase gene family of Brassica napus in response to Leptosphaeria maculans. Int. J. Mol. Sci. 19:3769.

Liu, H., Shao, B., Long, X., Yao, Y., and Meng, Q. 2016. Foliar penetration enhanced by biosurfactant rhamnolipid. Colloids Surf. B Biointerfaces 145:548-554.

Marcroft, S. J., Van de Wouw, A. P., Salisbury, P. A., Potter, T. D., and Howlett, B. J. 2012. Effect of rotation of canola (Brassica napus) cultivars with different complements of blackleg resistance genes on disease severity. Plant Pathol. 61:934-944.
Mohan, P. K., Nakhla, G., and Yanful, E. K. 2006. Biokinetics of biodegradation of surfactants under aerobic, anoxic and anaerobic conditions. Water Res. 40:533-540.

Monnier, N., Furlan, A., Botcazon, C., Dahi, A., Mongelard, G., Cordelier, S., Clement, C., Dorey, S., Sarazin, C., and Rippa, S. 2018. Rhamnolipids from Pseudomonas aeruginosa are elicitors triggering Brassica napus protection against Botrytis cinerea without physiological disorders. Front. Plant Sci. 9: 1170.

Monnier, N., Furlan, A. L., Buchoux, S., Deleu, M., Dauchez, M., Rippa, S., and Sarazin, C. 2019. Exploring the dual interaction of natural rhamnolipids with plant and fungal biomimetic plasma membranes through biophysical studies. Int. J. Mol. Sci. 20:1009.

Oliver, R. 2012. Genomic tillage and the harvest of fungal phytopathogens. New Phytol. 196:1015-1023.

Özdemir, G., Peker, S., and Helvaci, S. S. 2004. Effect of pH on the surface and interfacial behavior of rhamnolipids R1 and R2. Colloids Surf. A Physicochem. Eng. Asp. 234:135-143.

Pongam, P., Osborn, T. C., and Williams, P. H. 1998. Genetic analysis and identification of amplified fragment length polymorphism markers linked to the alm1 avirulence gene of Leptosphaeria maculans. Phytopathology 88: 1068-1072.

R Core Team. 2014. R: A Language and Environment for Statistical Computing. R Foundation for Statistical Computing, Vienna, Austria.

Rikalovic, M. G., Abdel-Mawgoud, A. M., Déziel, E., Gojgic-Cvijovic, G. D., Nestorovic, Z., Vrvic, M. M., and Karadzic, I. M. 2013. Comparative analysis of rhamnolipids from novel environmental isolates of Pseudomonas aeruginosa. J. Surfactants Deterg. 16:673-682.

Rikalović, M. G., Vrvić, M. M., and Karadžić, I. M. 2015. Rhamnolipid biosurfactant from Pseudomonas aeruginosa-From discovery to application in contemporary technology. J. Serb. Chem. Soc. 80:279-304.

Sanchez, L., Courteaux, B., Hubert, J., Kauffmann, S., Renault, J.-H., Clément, C., Baillieul, F., and Dorey, S. 2012. Rhamnolipids elicit defense responses and induce disease resistance against biotrophic, hemibiotrophic, and necrotrophic pathogens that require different signaling pathways in Arabidopsis and highlight a central role for salicylic acid. Plant Physiol. 160:1630-1641.

Sewell, T. R., Hawkins, N. J., Stotz, H. U., Huang, Y., Kelly, S. L., Kelly, D. E., Fraaije, B., and Fitt, B. D. L. 2017. Azole sensitivity in Leptosphaeria pathogens of oilseed rape: The role of lanosterol $14 \alpha$-demethylase. Sci. Rep. 7:15849.

Sha, R., Jiang, L., Meng, Q., Zhang, G., and Song, Z. 2012. Producing cellfree culture broth of rhamnolipids as a cost-effective fungicide against plant pathogens. J. Basic Microbiol. 52:458-466.

Sonah, H., Zhang, X., Deshmukh, R. K., Borhan, M. H., Fernando, W. G. D., and Bélanger, R. R. 2016. comparative transcriptomic analysis of virulence factors in Leptosphaeria maculans during compatible and incompatible interactions with canola. Front. Plant Sci. 7:1784.

Sprague, S. J., Balesdent, M.-H., Brun, H., Hayden, H. L., Marcroft, S. J., Pinochet, X., Rouxel, T., and Howlett, B. J. 2006. Major gene resistance in Brassica napus (oilseed rape) is overcome by changes in virulence of populations of Leptosphaeria maculans in France and Australia. Eur. J. Plant Pathol. 114:33-40.

Stanghellini, M. E., and Miller, R. M. 1997. Biosurfactants: Their identity and potential efficacy in the biological control of zoosporic plant pathogens. Plant Dis. 81:4-12.

Tan, Y. N., and Li, Q. 2018. Microbial production of rhamnolipids using sugars as carbon sources. Microbiol. Cell Fact. 17:89.

Thomma, B. P. H. J., Eggermont, K., Penninckx, I. A. M. A., Mauch-Mani, B., Vogelsang, R., Cammue, B. P. A., and Broekaert, W. F. 1998. Separate jasmonate-dependent and salicylate-dependent defense-response pathways in Arabidopsis are essential for resistance to distinct microbial pathogens. Plant Biol. 95:15107-15111.

Varnier, A. L., Sanchez, L., Vatsa, P., Boudesocque, L., Garcia-Brugger, A., Rabenoelina, F., Sorokin, A., Renault, J.-H., Kauffmann, S., Pugin, A., Clement, C., Baillieul, F., and Dorey, S. 2009. Bacterial rhamnolipids are novel MAMPs conferring resistance to Botrytis cinerea in grapevine. Plant Cell Environ. 32:178-193.

Wang, Z., Fang, H., Chen, Y., Chen, K., Li, G., Gu, S., and Tan, X. 2014. Overexpression of BnWRKY33 in oilseed rape enhances resistance to Sclerotinia sclerotiorum. Mol. Plant Pathol. 15:677-689.

West, J. S. J., Kharbanda, P. D., Barbetti, M. J. M., and Fitt, B. D. L. 2001. Epidemiology and management of Leptosphaeria maculans (Phoma stem canker) on oilseed rape in Australia, Canada and Europe. Plant Pathol. 50: $10-27$. 